\title{
Condition Monitoring of Turbogenerator by Vibration Monitoring Technique
}

\author{
Manoj Kumar Chittora \\ Sr. Lecturer (Mech.), Govt. Polytechnic College, Kota (Rajasthan)
}

\begin{abstract}
The new generation of condition monitoring and diagnostics systems plays an important role in efficient functioning of thermal power plants. Most rotating machine defects can be detected by such a system much before dangerous situation occurs. It allows the efficient use of stationary on-line continuous monitoring system for condition monitoring and diagnostics as well. Vibration monitoring for condition monitoring of turbine bearing can reduce expenses of maintenance of turbo generator in power plant as well as prevent unnecessary shut down of plant, which create the power crisis. Vibration monitoring system of turbo generator is based on the vibration signature analysis as well as manual vibration data analysis with the help of mechanalysis severity chart. The vibration signature as well as manual data is analyzed from two parameters, namely vibration amplitude (displacement \& velocity) of bearing casing and absolute shaft vibration at each bearing. The object of this paper is to giving brief idea about measuring vibration amplitude (manually as well as vibration signature) and analyzing method of data obtained, identification procedure of problems \& suggesting a corrective action, associated with unit 3-turbo generator system of super thermal power station. In This paper author also suggested some latest techniques of vibration monitoring \& analysis.
\end{abstract}

Keywords-vibration ,signature ,amplitude, frequency, load.

\section{INTRODUCTION}

Due to deficiency of power in country these days power generation industries are gaining more \& more attention of Govt. Management of power plants are also wants to utilize full plant capacity with high efficiency so that per unit cost of production could be minimized. This can be achieved by reducing failure of various components of plant and reducing heat loss, which are only possible when maintenance and operation schedule of plant prepared scientifically. In past, industry prepare the maintenance schedule by past experience, but these days maintenance schedule is prepared by experience as well as according to the condition of equipment, which is measured regularly by monitoring the certain parameters.

In power plant, measurement of the amplitude, velocity, and frequency of vibration are taken at each bearing of turbine \& generator. Except these for complete monitoring the condition of turbo generator unit of any power plant following parameters are also generally measured at regular interval of time or on line continuously [6,7]: -

(1) Load (MW) [2] Frequency [Hz] (3) MVAR / Cos $\phi$ (4) Winding temperature(5) Core temperature (6) Hydrogen temperature (7) Vibration Frequency- Horizontal vertical and Axial (8) Lube Oil temperature (9) Shell pressure(10) LP exhaust hood temperature (11) Condenser vacuum (12) Super heater \& preheated temperature (13) Bearing temperatures (14) Shaft vibrations.

After measuring these parameters, comparison is done with standard value set by manufacturer of Equipment or by management or sdandered set by agency authorize internationally. If there are certain abrupt results occur than maintenance is carried out. Thus predictive maintenance programme or condition monitoring of various equipments not only reduce unscheduled down time due to breakdown of components but also proves to be economical as unnecessary dismantling and replacements are avoided.

\section{VIBRATION MONITORING TECHNIQUES}

Following Techniques [12] are generally used for obtaining vibration data for the purpose of detecting and Identifying specific problem in rotating Machinery:-

(a) Manually by using transducers

(b) Machine signature by using different IRD analyzers in the following pattern

- Amplitude versus frequency

- $\quad$ Amplitude versus time.

- Amplitude versus frequency versus time

- $\quad$ Time wave form

- $\quad$ Lissajous Patterns (Orbits)

- $\quad$ Amplitude and Phase Versus RPM

- $\quad$ Phase versus time

- $\quad$ Mode shape determination

A thorough evaluation of Machine history \& its characteristics, gives us the various information, which are very much helpful in selecting the analysis procedure. At thermal power station, for turbo generator, vibration data are obtained by IRD 880 Analyzer in the form of amplitude (displacement \& velocity) versus frequency for analysis purpose manually as well as in the graphical form (machine signature). 


\section{OBSERVATIONS}

Table 1.1 shows the vibration observations taken manually \& figure 1.1 shows the machine vibration signature of bearing no 1 of a turbo generator of thermal power station for year 2011.

Table 1.1 Vibration observations

\begin{tabular}{|c|c|c|c|c|c|}
\hline DATE/ TIME & $\begin{array}{l}13 / 1 / 11 \\
11.20 \\
\text { AM }\end{array}$ & $\begin{array}{l}\text { 27/1/11 } \\
11.30 \\
\text { AM }\end{array}$ & $\begin{array}{l}\text { 2/2/11 } \\
11: 00 \\
\text { AM }\end{array}$ & $\begin{array}{l}\text { 18/2/11 } \\
\text { 11:05 AM }\end{array}$ & $\begin{array}{l}\text { 3/03/11 } \\
\text { 11: 00 AM }\end{array}$ \\
\hline LOAD (MW) & 202 & 211 & 209 & 211 & 209 \\
\hline FREQ(Hz) & 48.93 & 49.19 & 48.63 & 48.88 & 48.95 \\
\hline VIBRATION & - & - & - & - & - \\
\hline $\begin{array}{l}\text { Brg.I Vertical micron } / \mathrm{mm} / \\
\text { sec }\end{array}$ & $24 / 4.7$ & $23 / 4.4$ & $28 / 5.5$ & $28 / 5.5$ & $22 / 5.5$ \\
\hline $\mathrm{H}$ (Horizontal) & $38 / 5.8$ & $42 / 6.7$ & $41 / 6.6$ & $36 / 6.7$ & $30 / 5.3$ \\
\hline A (Axial) & $44 / 7.0$ & $34 / 5.8$ & $40 / 6.6$ & $33 / 5.1$ & $8 / 3$ \\
\hline Absolute shaft vibration & 143 & 163 & 157 & 161 & 155 \\
\hline
\end{tabular}

\begin{tabular}{|c|c|c|c|c|c|}
\hline DATE/ TIME & $\begin{array}{l}\text { 17/03/11 } \\
11.00 \\
\text { AM }\end{array}$ & $\begin{array}{l}7 / 4 / 11 \\
11: 40 \\
\text { AM }\end{array}$ & $\begin{array}{l}\text { 24/05/11 } \\
11: 45 \text { AM }\end{array}$ & $\begin{array}{l}\text { 5/7/11 } \\
11: 00 A M\end{array}$ & $\begin{array}{l}\text { 17/7/11 } \\
\text { 11:05 AM }\end{array}$ \\
\hline LOAD (MW) & 210 & 207 & 209 & 205 & 209 \\
\hline FREQ $(\mathrm{Hz})$ & 49.85 & 48.13 & 49.65 & 48.92 & 48.9 \\
\hline VIBRATION & - & - & - & - & - \\
\hline $1 \mathrm{~V}$ micron $/ \mathrm{mm} / \mathrm{sec}$ & $23 / 5.6$ & $26 / 5.8$ & $22.4 / 5.4$ & $20 / 2.9$ & $20.5 / 4.7$ \\
\hline $\mathrm{H}$ & $35 / 6.2$ & $33 / 5.8$ & $28.2 / 5.3$ & $27.2 / 4.8$ & $29.1 / 5.1$ \\
\hline $\mathrm{A}$ & $29 / 5.3$ & $15 / 3.5$ & $8.1 / 3.6$ & $16 / 3.1$ & $17 / 3.4$ \\
\hline Shaft Vib.of brg.1 & 153 & 171 & $153-$ & 137 & 144 \\
\hline
\end{tabular}

\begin{tabular}{|l|l|l|l|l|l|}
\hline DATE/ TIME & $\mathbf{1 / 8 / 1 1}$ & $\mathbf{2 1 / 8 / 1 1}$ & $\mathbf{1 1 / 1 0 / 1 1}$ & $\mathbf{2 6 / 1 0 / 1 1}$ & $\mathbf{6 / 1 1 / 1 1}$ \\
& $\mathbf{1 1 : 4 8 A M}$ & $\mathbf{1 0 : 5 0} \mathbf{A M}$ & $\mathbf{4 . 0 0} \mathbf{~ p m}$ & $\mathbf{3 . 3 0}$ AM & $\mathbf{1 1}$ : $\mathbf{A M}$ \\
\hline LOAD $(\mathrm{MW})$ & 206 & 210 & 207 & 212 & 211 \\
\hline FREQ(Hz) & 48.03 & 49.33 & 49.04 & 49.21 & 49.15 \\
\hline VIBRATION & - & - & - & - & - \\
\hline IV micron /mm/ sec & $28.5 / 5.6$ & $24 / 4.8$ & $24 / 5.1$ & $19 / 4.8$ & $18 / 4.8$ \\
\hline $\mathrm{H}$ & $3.56 / 5.8$ & $8.5 / 3$ & $10 / 3.4$ & $11 / 2.2$ & $11 / 2.6$ \\
\hline A & $20.5 / 3.9$ & $29 / 5.6$ & $13 / 3.1$ & $23 / 4$ & $27 / 4.5$ \\
\hline $\begin{array}{l}\text { Absolute Shaft Vib.of } \\
\text { brg 1 }\end{array}$ & 143 & 113 & 135 & 106 & 101 \\
\hline
\end{tabular}

FIGURE 1.1-machine signature

\begin{tabular}{|c|c|c|}
\hline $10-01-11$ & $10-01-11$ & $10-01-11$ \\
$U \neq 3$ & $U \neq 3$ & $U \neq 3$ \\
$1 V / D i s p$ & $1 \mathrm{H} / \mathrm{Disp}$ & $1 \mathrm{~A} / \mathrm{Disp}$ \\
$210 \mathrm{MW} / 48.66 \mathrm{~Hz}$ & $210 \mathrm{MW} / 48.66 \mathrm{~Hz}$ & $210 \mathrm{MW} / 48.66 \mathrm{~Hz}$ \\
$27(25-27)$ micron & $37(36-38)$ micron & $53(53-55)$ micron \\
\hline
\end{tabular}




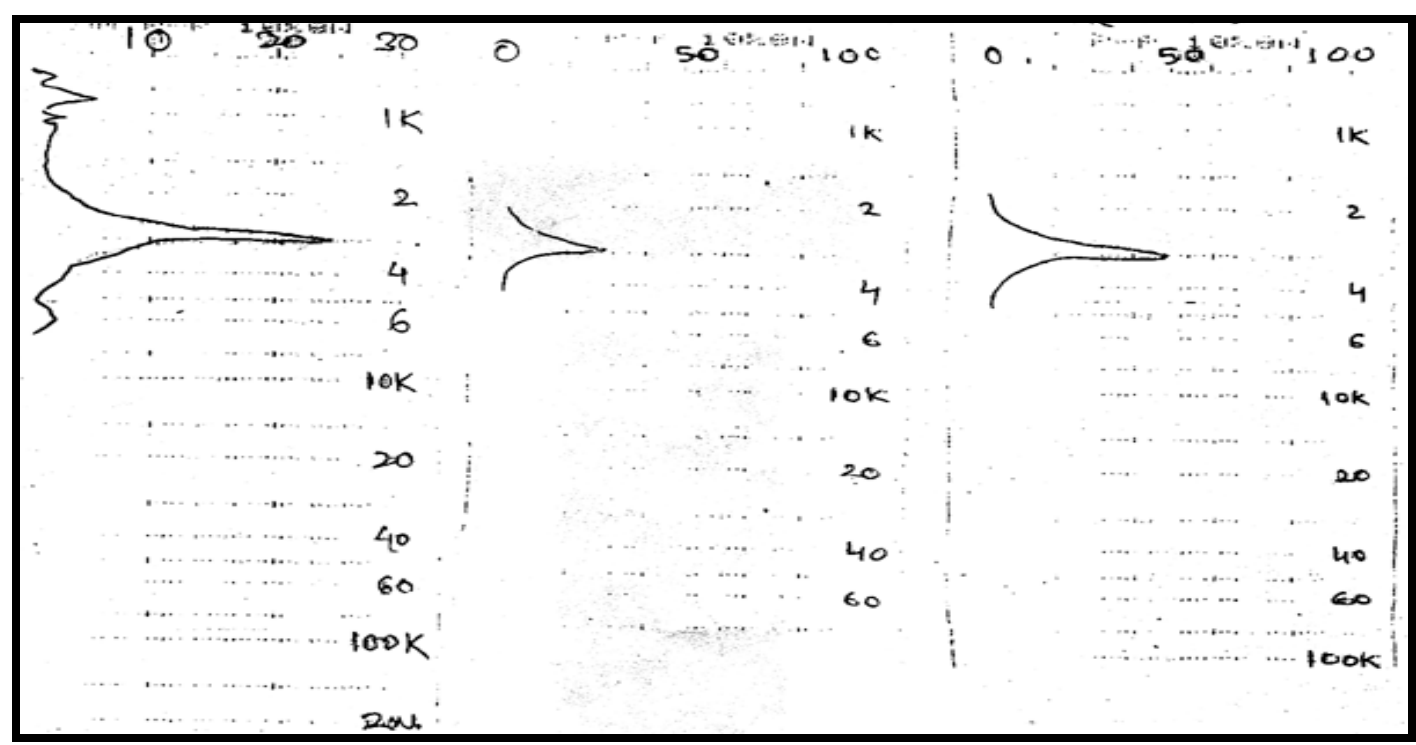

Figure1.2-machine signature

\begin{tabular}{|c|c|c|}
\hline $\begin{array}{c}10-01-11 \\
U \neq 3\end{array}$ & $10-01-11$ & $10-01-11$ \\
$\mathbf{U} \neq 3$ & $1 \mathrm{U} / \mathrm{Vel}$ & $1 \mathrm{~A} / \mathrm{Vel}$ \\
$1 \mathrm{~V} / \mathrm{Vel}$ & $210 \mathrm{MW} / 48.66 \mathrm{~Hz}$ & $210 \mathrm{MW} / 48.66 \mathrm{~Hz}$ \\
$210 \mathrm{MW} / 48.66 \mathrm{~Hz}$ & $\mathbf{5 . 6}(5.4-5.7) \mathrm{mm} / \mathrm{sec}$. & $8.7(8.6-8.9) \mathrm{mm} / \mathrm{sec}$. \\
$5.1(4.9-5.2) \mathrm{mm} / \mathrm{sec}$. &
\end{tabular}

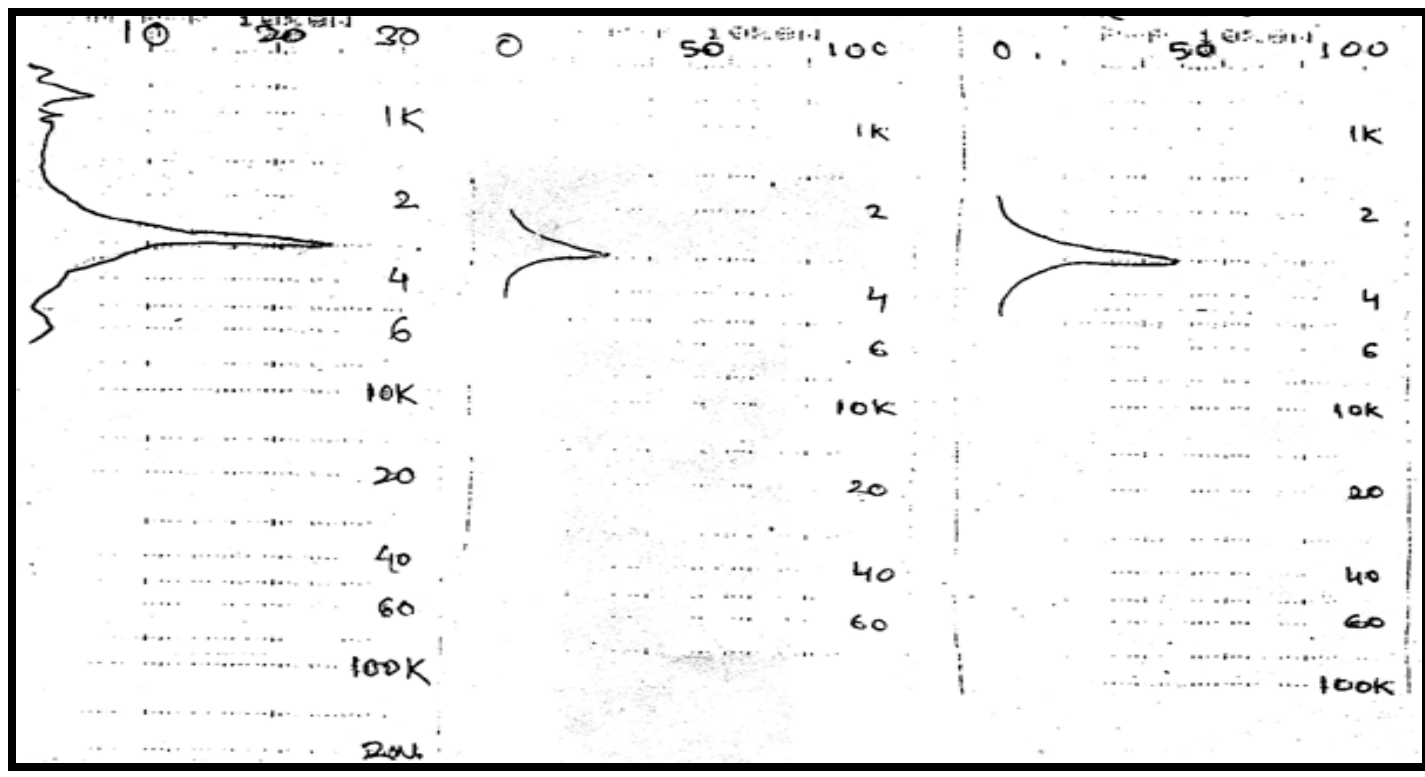

\section{ANALYSIS OF DATAS}

For analysis of vibration data obtained, analysis tables were prepared for each bearing by taking manual vibration amplitude, load \& frequency, machine signature values with Date \& time. For analysis of problems, these data's and signature were compared with the standard data's. By using IRD mechanalysis severity chart for abrupt values, problems were identified. Standard values for different parameters are as follows which are useful for analysis papoose:-

Radial vibration displacement -100 micron peak to peak unit shut down limit.

35 micron rms value alarming limit.

50 micron peak to beak alarming limit.

Radial Vibration velocity $6.4 \mathrm{~mm} / \mathrm{Sec}$. rms value

- $8 \mathrm{~mm} / \mathrm{sec}$. peak to peak

Absolute shaft vibration 120 micron peak to peak alarming limit 200 micron peak to peak Unit shut down limit 
Axial vibration amplitude $\quad-\quad 1 / 2$ of highest radial vibration

(displacement \& Velocity) i.e. 25 micron alarming limit.

\section{1- Analysis Tables}

For analysis purpose, table is prepared for bearing no.1 for H.P. turbine as follows;

Unit 3 - Bearing No- 1-

Table No. 1.2

\begin{tabular}{|c|c|c|c|c|c|c|c|c|c|}
\hline \multirow{3}{*}{$\begin{array}{l}\mathbf{S .} \\
\mathbf{N}\end{array}$} & \multirow{3}{*}{$\begin{array}{l}\text { Date } \\
\text { with } \\
\text { Time }\end{array}$} & \multirow{3}{*}{$\begin{array}{l}\text { Mode of } \\
\text { Measure- } \\
\text { ment }\end{array}$} & \multirow{3}{*}{$\begin{array}{l}\text { Load } \\
(M w) \\
\& \\
\text { f(Hz) } \\
\end{array}$} & \multicolumn{6}{|c|}{ Amplitude Measurement } \\
\hline & & & & \multicolumn{2}{|l|}{ Vertical } & \multicolumn{2}{|c|}{ Horizontal } & \multicolumn{2}{|l|}{ Axial } \\
\hline & & & & $\begin{array}{l}\text { Displace- } \\
\text { ment in } \\
\text { micron }\end{array}$ & $\begin{array}{l}\text { Velocity } \\
\text { in } \\
\mathrm{mm} / \mathrm{Sec}\end{array}$ & $\begin{array}{l}\text { Displa- } \\
\text { cement }\end{array}$ & Velocity & $\begin{array}{l}\text { Displa- } \\
\text { Cement }\end{array}$ & Velocity \\
\hline 1 & $\begin{array}{c}\text { 3/1/11 } \\
11 \text { A.M }\end{array}$ & $\mathrm{M}$ & $\begin{array}{c}202 \\
48.93\end{array}$ & 24 & 4.7 & 35 & 5.8 & 44 & 7.0 \\
\hline 2. & $\begin{array}{l}\text { 18/1/11 } \\
\text { 11 A.M }\end{array}$ & $\mathrm{M}$ & $\begin{array}{c}211 \\
49.19\end{array}$ & 23 & 4.4 & 42 & 6.7 & 34 & 5.8 \\
\hline 3. & $10 / 1 / 11$ & MS & & 27 & 5.1 & 37 & 5.6 & 53 & 8.7 \\
\hline 4. & $\begin{array}{c}\text { 2/2/11 } \\
11 \text { A.M }\end{array}$ & $\mathrm{M}$ & $\begin{array}{c}209 \\
48.63\end{array}$ & 28 & 5.5 & 41 & 6.6 & 40 & 6.6 \\
\hline 5. & $\begin{array}{c}18 / 2 / 11 \\
11: 02 \mathrm{AM}\end{array}$ & $\mathrm{M}$ & $\begin{array}{c}211 \\
48.88\end{array}$ & 28 & 5.5 & 36 & 6.7 & 33 & 5.1 \\
\hline 6. & $\begin{array}{c}3 / 3 / 11 \\
11 \mathrm{AM}\end{array}$ & $\mathrm{M}$ & $\begin{array}{c}209 \\
48.95\end{array}$ & 22 & 5.5 & 30 & 5.3 & 8 & 3 \\
\hline 7. & $\begin{array}{c}17 / 3 / 11 \\
11: 10 \mathrm{AM}\end{array}$ & $\mathrm{M}$ & $\begin{array}{c}210 \\
49.85\end{array}$ & 23 & 5.6 & 35 & 6.2 & 12 & 3.2 \\
\hline 8. & $\begin{array}{c}7 / 4 / 11 \\
11: 40 \mathrm{AM}\end{array}$ & $\mathrm{M}$ & $\begin{array}{c}207 \\
48.13\end{array}$ & 26 & 5.8 & 33 & 5.8 & 15 & 3.5 \\
\hline 9 & $\begin{array}{l}24 / 5 / 11 \\
11: 45 \text { AM }\end{array}$ & $\mathrm{M}$ & $\begin{array}{c}209 \\
49.65\end{array}$ & 22.4 & 5.4 & 28.2 & 5.3 & 8.1 & 3.6 \\
\hline 10 & $25 / 5 / 11$ & MS & $\begin{array}{c}209 \\
49.65\end{array}$ & 28 & 6.2 & 31 & 5.7 & 13 & 3.5 \\
\hline 11 & $\begin{array}{l}5 / 7 / 11 \\
11 \mathrm{AM}\end{array}$ & $\mathrm{M}$ & $\begin{array}{c}205 \\
48.92\end{array}$ & 20 & 4.1 & 27.2 & 4.8 & 16 & 3.1 \\
\hline 12 & $5 / 7 / 11$ & MS & & 20 & 4.1 & 27 & 4.8 & 16 & 3.1 \\
\hline 13 & $\begin{array}{l}17 / 7 / 11 \\
11: 03 \mathrm{AM}\end{array}$ & $\mathrm{M}$ & $\begin{array}{c}208 \\
48.89\end{array}$ & 20.5 & 4.7 & 29 & 5.1 & 17 & 3.4 \\
\hline 14 & $\begin{array}{l}\text { 1/8/11 } \\
11: 48 \mathrm{AM}\end{array}$ & $\mathrm{M}$ & $\begin{array}{c}206 \\
48.03\end{array}$ & 28.5 & 5.6 & 35.6 & 5.8 & 20.5 & 3.9 \\
\hline 15 & $\begin{array}{l}21 / 8 / 11 \\
10: 50 \mathrm{AM}\end{array}$ & $\mathrm{M}$ & $\begin{array}{c}210 \\
49.33\end{array}$ & 24 & 4.8 & 8.5 & 3 & 29 & 5.6 \\
\hline 16 & $21 / 8 / 11$ & MS & & 24 & 4.8 & 8.5 & 3 & 29 & 5.6 \\
\hline 17 & $\begin{array}{l}11 / 10 / 11 \\
4 \mathrm{PM}\end{array}$ & $\mathrm{M}$ & $\begin{array}{l}207 \\
49.21\end{array}$ & 24 & 5.1 & 10 & 3.4 & 13 & 3.1 \\
\hline 18 & $\begin{array}{l}26 / 10 / 11 \\
3.30 \mathrm{PM}\end{array}$ & $\mathrm{M}$ & $\begin{array}{l}212 \\
49.21\end{array}$ & 19 & 4.8 & 11 & 2.2 & 23 & 4 \\
\hline 19 & $\begin{array}{l}\text { 6/11/11 } \\
\text { 11:10 AM }\end{array}$ & $\mathrm{M}$ & $\begin{array}{l}211 \\
49.15\end{array}$ & 18 & 4.8 & 11 & 2.6 & 27 & 4.5 \\
\hline
\end{tabular}

Analysis \& Conclusion

1- For this bearing as axial vibration are higher than Radial vibration in machine signature taken on 10/1/11 as well vibration amplitude taken by IRD 880 manually from dated 3/1/11 to 18/2/11 as shown in analysis table1.2. On dated 18/2/11 axial vibration were 33 micron which were higher than radial vibration amplitude, which was in alarming limit in case of axial vibrations as these must be half ( 25 micron) of radial vibration (50 micron).By observing machine signature and comparing with standard one, it is observed that peak occurs at 1x RPM so with the help of severity chart it can be concluded that this is a case of misalignment or bent shaft.

2. Another observation with machine signature taken on $10 / 1 / 11$ was that in case of vertical displacement there are disturbances at the starting which shows oil whirl problem and instability of shaft. 
3. From observation, it can be seen that absolute shaft vibration of this bearing are continuously increasing above the alarming limit (120 micron) which show that there are some unbalance forces coming on rotor shaft. From machine signature \& manual readings taken by IRD 880, we observed that Axial vibration of Ist bearing are high from 3/1/11 to $18 / 2 / 11$ as well absolute shaft vibration are high which shows that this is a case of rotor unbalance.

Corrective action- By taking 1 day shutdown, these problems were rectified by aligning rotor properly with shaft \& bearing and adjusting the pressure of lubricant as per manufacturer recommendation as well as by tightening the foundation bolt properlymay removes the problem of misalignment and oil whirl \& looseness of foundation.

After this treatment, axial vibration came within limit ( 8 micron on dated 3/3/11) as oil whirl problem was shorted out as shown in machine signature taken on dated 25/5/11, but absolute shaft vibration were coming high (above alarming limit).From severity chart (IRD Mechanalyis, vibration \& noise identification chart) we can say this was a problem of unbalance Rotor of HP turbine as bearing 1 situated at Front plane of HPT.

For rectifying the problems a major shut down was taken on dated 25/5/11 and complete over hauling of unit was done as well as try to balance the rotor. After assembling \& Running the unit on Load on dated 5/7/11, observation shows that absolute shaft vibration (137 Micron) were still on higher side and showing increasing trend. Again a shutdown was taken on 20/8/11 \& balancing of Rotor was done by BHEL people which detail is given with bearing no. 3. After this absolute shaft vibration value on dated 21/8/11 were 113 micron which was under alarming limit.

4. After balancing, signature for bearing 1 taken on 21/8/11 for displacement, as well velocity were completely disturbed. BHEL people checked bearing lubrication flow (pressure \& temperature), checked steam flow \& adjusted as per manufacturer requirement Bearing housing was tighten Rigidly and Machine damping was increased.

5. After balancing, vertical amplitude as well velocity were coming higher than Horizontal amplitude readings but within limit unto dated 6/11/11. But after this observation from dated 17/11/11 show that vertical amplitude were more than 2 times higher than Horizontal amplitude as well as axial vibrations are coming on higher side. Thus with the help of severity chart, it can be concluded that this is a case of mechanical looseness \& misalignment.

\section{OVERALL CONCLUSION}

From analysis of observations taken for bearing no no1 and conclusion regarding problems of bearing nolof unit 3 turbo generator, finally it could be concluded that Rotor unbalance, misalignment of shaft of turbine and generator, oil whirl were the general problems occurred during year2011. These problems were rectified by taking shutdown of unit for overhauling and balancing the rotor of low pressure turbine, improving damping of generator foundation as discussed with each bearing.

It is evident from above discussion that vibration-monitoring technique is very powerful tool for assessing the condition of rotary machine and if used with wisdom it can reduce the breakdowns of power plants. Thus we can say that the use of this technique is even more desirable these days with increasing gap in demand and supply of power.

\section{REFERENCES}

[1]. Anand M.M.S. [2004“Electronic Instruments \& Instrumentation Technology” Prentic Hall of India private limited PP 29.

[2]. Bandyopadhyay Ashok, Mandal Shyamal Kr. Das, Barnali Pal [2004] "Real time condition Monitoring system using vibration analysis for turbine bearing" speech \& signal processing group, ER \& DCI Calcutta PP 1-4.

[3]. Bhadury Dr. Bikas \& Basu Dr. S.K. [2004] "Reliability Engineering \& Maintenance Management" PP 47-51.

[4]. Bhadury Dr. Bikas \& Basu Dr. S.K. [2004] "Reliability Engineering \& Maintenance Management” PP 223-248.

[5]. CSIO [2005] "Data Acquisition system \& Instrumentation" Chandigraph PP 2-10.

[6]. EPRI [2002] "Power Industry vision for predictive Maintenance" Turbine system conference Feb 25-26, 2002 PP $2-7$.

[7]. Mechanlysis [2005] "Advanced training manual of IRD 880 for vibration measurement” PPI-37.

[8]. Mechanalysis [2005] “Advanced training manual of IRD 880 for vibration analysis" PP1-3, 86-114.

[9]. Operation Manual 28838 of IRD Mechanlysis Model 880 spectrum analyzer PP- 11. 